\title{
Association Between Attention Deficit Hyperactivity Disorder and Risk of Burn Injury: A Propensity-Matched Cohort Study
}

This article was published in the following Dove Press journal: Neuropsychiatric Disease and Treatment

\author{
Jia-Yin Yeh (D) \\ Tsai-Yu Hou' \\ Wei-Ting Tseng (1D \\ Vincent Chin-Hung Chen ${ }^{2,3}$ \\ Yao-Hsu Yang (iD ${ }^{4,5}$ \\ Ting-Yu Kuo ${ }^{4}$ \\ Jun-Cheng Weng ${ }^{6,7}$ \\ Charles Tzu-Chi Lee (iD ${ }^{8}$ \\ Yi-Lung Chen (1D) 9,10 \\ Min-Jing Lee $\mathbb{1}^{2,3}$ \\ 'Department of Psychiatry, Chang Gung \\ Memorial Hospital, Kaohsiung Branch, \\ Kaohsiung, Taiwan; ${ }^{2}$ Department of \\ Psychiatry, Chang Gung Memorial Hospital, \\ Chiayi Branch, Chiayi, Taiwan; ${ }^{3}$ School of \\ Medicine, Chang Gung University, Taoyuan, \\ Taiwan; ${ }^{4}$ Health Information and \\ Epidemiology Laboratory, Chang Gung \\ Memorial Hospital, Chiayi Branch, Chiayi, \\ Taiwan; ${ }^{5}$ Department of Traditional Chinese \\ Medicine, Chang Gung Memorial Hospital, \\ Chiayi Branch, Chiayi, Taiwan; ' ${ }^{6}$ Department \\ of Medical Imaging and Radiological Sciences, \\ Chang Gung University, Taoyuan, Taiwan; \\ ${ }^{7}$ Medical Imaging Research Center, Institute \\ for Radiological Research, Chang Gung \\ University and Chang Gung Memorial \\ Hospital at Linkou, Taoyuan, Taiwan; \\ ${ }^{8}$ Department of Health Promotion and \\ Health Education, National Taiwan Normal \\ University, Taipei, Taiwan; ${ }^{9}$ Department of \\ Healthcare Administration, Asia University, \\ Taichung, Taiwan; ${ }^{10}$ Department of \\ Psychology, Asia University, Taichung, \\ Taiwan
}

Correspondence: Min-Jing Lee Department of Psychiatry, Chang Gung Memorial Hospital, Chiayi, Taiwan, No. 6 ,

W. Sec., Jiapu Road, Puzi City, Chiayi

County 613, Taiwan

Tel +886-5-3621000 ext2303

Fax +886-5-3622188

Email 8035c@cgmh.org.tw
Purpose: Literature suggests that attention deficit hyperactivity disorder (ADHD) is associated with a high risk of unintentional injury. However, few studies have focused on whether risk of burn injury is relatively high among patients with ADHD. The aim of this study was to investigate whether ADHD affects the risk of burn injury.

Materials and Methods: Individuals aged $<18$ years with a current diagnosis of ADHD (N $=52,705)$ and age-, sex-, and other comorbidity-matched controls were selected from Taiwan's National Health Insurance Research Database for the period of January 1996 to December 2013. Burn injury was identified in both groups, and risk was evaluated using Cox proportional hazards models. We also explored the effects of age and sex on the association. Results: We determined that patients with ADHD had an increased probability of burn injury compared with the control group (ADHD vs controls, $4.6 \%$ vs $2.6 \%$; adjusted hazard ratio $[\mathrm{aHR}]=1.78 ; 95 \%$ confidence interval $[\mathrm{CI}]=1.66-1.90)$. The effect of ADHD on burn injury was more prominent among those aged $<6$ years $(\mathrm{aHR}=1.96 ; 95 \% \mathrm{CI}=1.75-2.20)$ relative to those aged $\geq 6$ years $(\mathrm{aHR}=1.69 ; 95 \% \mathrm{CI}=1.56-1.83)$. Both sexes had similar risk profiles.

Conclusion: The study findings contribute to the increasing body of evidence that ADHD is associated with proneness to burn injury, particularly in children aged $<6$ years.

Keywords: ADHD, burn injury, propensity-matched, cohort

\section{Introduction}

Attention deficit hyperactivity disorder (ADHD) is a common neurodevelopmental disorder with a global lifetime prevalence of approximately $7.2 \% .{ }^{1}$ ADHD is characterized by inattention, hyperactivity, and impulsivity. These symptoms are indicative of impairment of executive function. ${ }^{2}$ Several studies have revealed that ADHD is associated with injury proneness, ${ }^{3-8}$ and children with ADHD have more severe injuries and more often require hospital treatment following an injury than do those without ADHD. ${ }^{9,10}$ The incidence of injury results in the shortening of life expectancy and is one of the most common causes of death among children with ADHD. ${ }^{11,12}$ Among all injuries, burn injury is catastrophic and has clinical implications. Although burn injury is regarded as an acute event, its sequential effect on psychological and physical function can be comparable to that of a chronic condition. ${ }^{13,14}$

Several studies have investigated the association between ADHD and increased burn injury risk in children. ${ }^{15-19}$ One case-control study including 223 patients demonstrated that the prevalence of burns among children with ADHD (10.6\%) was 
considerably higher than that among the controls $(2.0 \%){ }^{19}$ Badger et al conducted a retrospective study and reported that children with ADHD were more frequently involved in high-risk activities leading to burn injury and that they more frequently experienced mental health problems. ${ }^{16}$ However, the literature contains limited data regarding the association between burn injury and ADHD in clinical settings under the consideration of other mental health problems and possible confounders.

Studies investigating ADHD and injury proneness through database analyses have reported burn injury risk among patients with ADHD. ${ }^{4-7,20}$ However, the results of these studies were inconclusive. A national cohort study conducted in the United Kingdom examined correlations between childhood psychiatric disorder and unintentional injury in more than 10,000 children aged 5-15 years. ${ }^{4}$ The results revealed that burn injury was more common among patients with ADHD than among those without diagnosed psychiatric conditions, but the statistical significance of the effect vanished after other psychiatric diseases were adjusted for. Another database study conducted in Germany identified no significant difference in burn risk between children with ADHD and those without. ${ }^{7}$ One population study conducted using claims data in Taiwan reported that patients with ADHD had a higher burn injury risk than did controls, with the corresponding odds ratio (OR) being 1.6. ${ }^{5}$ Individuals aged 6-18 years were included in the study; those aged younger than 5 years, who are at higher risk of burn injury, ${ }^{21,22}$ were not examined in the study. In addition to age, a wide range of potential confounders, such as sex, urbanization level of residence, medication use, and comorbidities, have been identified in evaluations of burn injury risk. ${ }^{18}$

Accordingly, we conducted this retrospective cohort study using a nationwide population-based database to comprehensively evaluate the risk of burn injury for children diagnosed as having ADHD, with possible confounders considered. We performed propensity score matching, in which the conditional probability of assignment to a particular group is based only on observed covariates, to balance the covariates of observational data. ${ }^{23}$ The use of propensity scoring with time-to-event data through weighting or matching techniques is well established. ${ }^{24}$

\section{Materials and Methods}

\section{Data Source}

This study was reviewed and approved by the Institutional Review Board of Chang Gung Memorial Hospital. The study used data from Taiwan's National Health Insurance Research Database (NHIRD) under the aegis of the National Health Research Institutes. Taiwan launched a single-payer National Health Insurance (NHI) program on March 1, 1995, covering all health care services for $99.5 \%$ of the national population. ${ }^{25}$ Because the NHI is a compulsory universal program for all residents in Taiwan, the NHIRD is a comprehensive health care database containing outpatient, ambulatory, hospital inpatient, and dental services data. The NHIRD also includes crucial information such as patients' demographic data, medical institutions visited, diagnostic codes, drugs prescribed, dates of all prescriptions, and all claimed medical expenses. The accuracy of disease diagnosis in the NHI system has been reported as being up to $99 \% .^{26,27}$ The database has been used in numerous epidemiologic studies. ${ }^{28,29}$

\section{Patient Selection and Propensity Score Matching}

Figure 1 illustrates the procedure employed for the selection of the study population. Individuals aged younger than 18 years receiving one or more inpatient or three or more outpatient diagnoses of ADHD (International Classification of Diseases, Ninth Revision, Clinical Modification [ICD9-CM] code 314) in 1 year between January 1, 1996, and December 31, 2013, were included in the ADHD cohort. We excluded individuals whose birth year was before 1996 $(\mathrm{n}=55,558)$ and those whose diagnosis occurred after 2012 ( $\mathrm{n}=9854$ ) because of insufficient duration of follow-up. To examine only incidence of burn injury following ADHD, patients diagnosed as having burn injury (ICD-9-CM codes 940-949) before a diagnosis of ADHD were also excluded. The dataset for the ADHD group finally contained data for 90,634 patients.

The control group was selected from the Longitudinal Health Insurance Database 2005 (LHID2005), a subset of the NHIRD, for which patients were randomly sampled from the 2005 Registry of Beneficiaries of the NHIRD. LHID2005 contains original claims data for 1 million individuals. Individuals not diagnosed as having ADHD during the monitoring period (January 1, 1996, to December 31, 2013) were enrolled as the control cohort. The control and ADHD groups were matched using 1:1 propensity score matching. Propensity scores were determined using multivariate logistic regression analysis with sex, age, urbanization level of residence, and comorbidities. Comorbidities associated with ADHD 


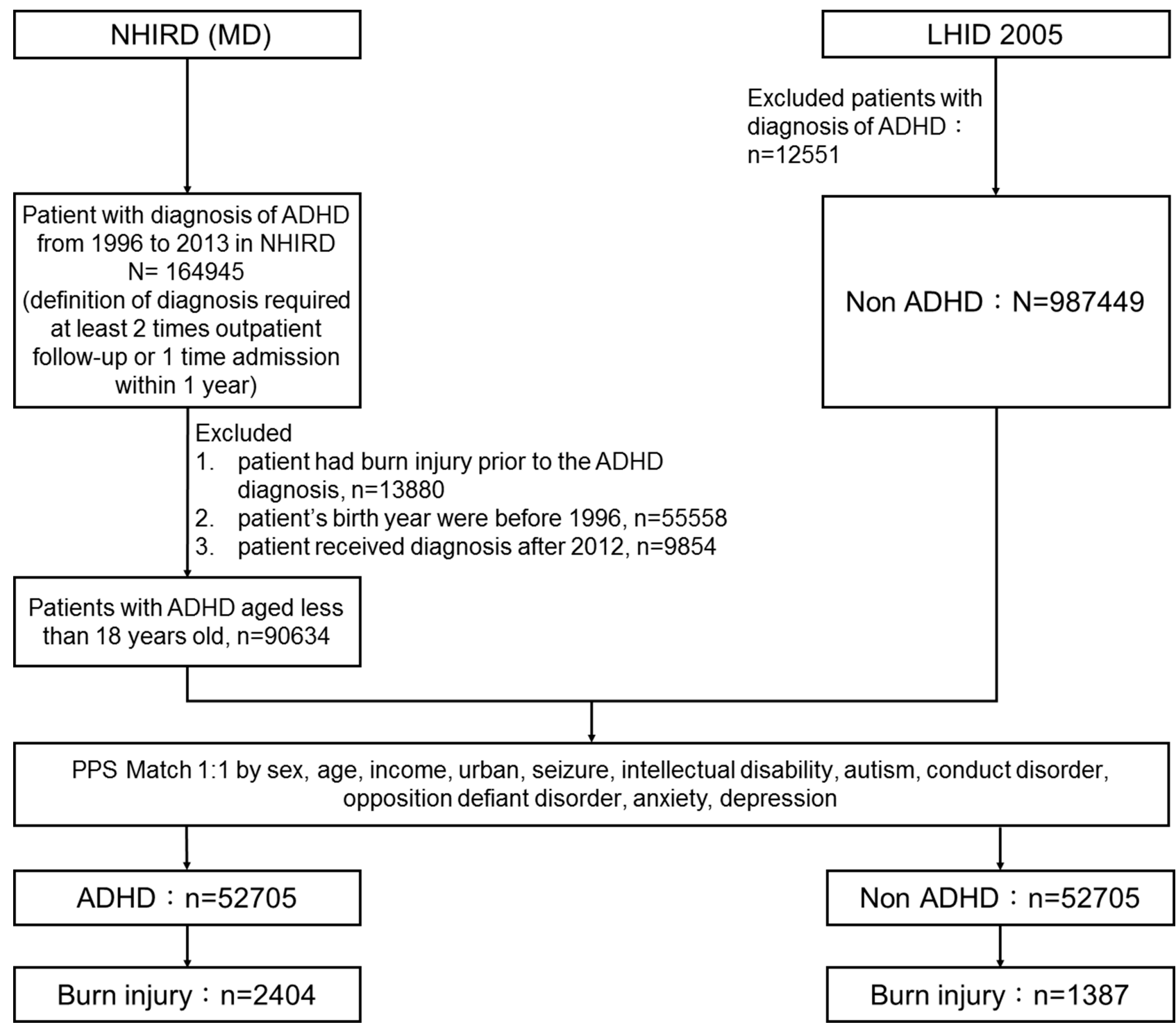

Figure I Flowchart for selection of the study population.

or burn injury including seizure (ICD-9-CM code 345), intellectual disability (ICD-9-CM codes 317-319), autism (ICD-9-CM code 299), conduct disorder (ICD-9-CM code 312), oppositional defiant disorder (ICD-9-CM code 313.81), anxiety (ICD-9-CM code 300), and depression (ICD-9-CM codes 296.2, 296.3, 300.4, and 311 ) were identified as confounding covariates. 5,30

\section{Outcome Measurement}

We defined incidence of burn injury (ICD-9-CM codes 940-949) as the outcome of interest. Each participant was followed up from the index date to the occurrence of the outcome, death, or the end of the study period (December 31, 2013). The index date was the date of first ADHD diagnosis. This date was matched in the control group to reduce potential temporal bias.

\section{Statistical Analysis}

All data management and statistical analysis processes were performed using SAS (version 9.3; SAS Institute Inc., Cary, $\mathrm{NC}$, USA). The chi-square $\left(\chi^{2}\right)$ test was used to compare characteristics between the ADHD and control groups and to determine the distribution of the study population. Variables are presented as numbers with percentages. A Cox proportional hazards regression model was employed to estimate burn injury risk for the ADHD group relative to the control group. A stratified Cox proportional hazards regression model was adopted for the subgroup analysis to evaluate 
the effects of age ( $\geq 6$ and $<6$ years) and sex (male and female). The results are presented as adjusted hazard ratios (aHRs) with 95\% confidence intervals (CIs).

\section{Results}

In total, 52,705 patients with ADHD and 1:1 propensity score matched non-ADHD controls were included in the study cohort. A comparison of the baseline characteristics of patients with ADHD with those of the non-ADHD controls is provided in Table 1. No difference was observed between the two groups in sex, age, urbanization level of residence, seizure, asthma, intellectual disability, autism, conduct disorder, oppositional defiant disorder, anxiety, or depression.

We evaluated the association between ADHD and burn injury. The incidence of burn injury in patients with ADHD was $4.6 \%$, higher than that of the non-ADHD controls $(2.6 \%$, standardized difference $=0.11)$. The influence of risk factors on burn injury was assessed using Cox proportional hazards regression analysis (Table 2).

We also performed a subgroup analysis using the stratified Cox proportional hazards regression model to investigate the impact of age ( $\geq 6$ and $<6$ years) and sex on burn injury (Table 3). The effect of ADHD on burn injury risk was more prominent among patients aged younger than 6 years $(\mathrm{aHR}=1.96 ; 95 \% \mathrm{CI}=1.75-2.20)$ than it was among those aged 6 years or older $(\mathrm{aHR}=1.69 ; 95 \% \mathrm{CI}=1.56-1.83)$. The sexes had a similar risk profile for burn injury.

\section{Discussion}

In this propensity-matched cohort study, we used a nationwide population database to evaluate the association between ADHD and burn injury. Compared with individuals in the non-ADHD group, patients with ADHD were significantly more prone to burn injury. The association between ADHD and burn injury was similar in individuals of either sex, but younger children were at higher risk of burn injury.

Previous studies have suggested that children with ADHD have a significantly higher risk of burn injury. One retrospective chart review revealed that the overall rate of ADHD diagnosis in children with burn injury was $13 \%$, which was higher than the prevalence in the general population. ${ }^{17}$ A case-control study comprising 123 patients with ADHD and 100 controls investigated the association between burn injury and ADHD. ${ }^{19}$ The results

Table I Comparison of Baseline Characteristics of Patients with ADHD and Non-ADHD Controls in the Propensity Model

\begin{tabular}{|c|c|c|c|c|c|}
\hline \multirow[t]{2}{*}{ Variables } & \multicolumn{2}{|c|}{ ADHD $(N=52,705)$} & \multicolumn{2}{|c|}{ Non-ADHD ( $N=52,705)$} & \multirow[t]{2}{*}{ Standardized Difference } \\
\hline & $\mathbf{N}$ & $\%$ & $\mathbf{N}$ & $\%$ & \\
\hline \multicolumn{6}{|l|}{ Gender } \\
\hline Female & 13,084 & 24.8 & 12,318 & 23.4 & 0.03 \\
\hline Male & 39,621 & 75.2 & 40,387 & 76.6 & -0.03 \\
\hline \multicolumn{6}{|l|}{ Age } \\
\hline $0-5$ & 9752 & 18.5 & 10,079 & 19.1 & -0.02 \\
\hline $6-11$ & 38,389 & 72.8 & 37,397 & 71 & 0.04 \\
\hline $12-18$ & 4564 & 8.7 & 5229 & 9.9 & -0.04 \\
\hline \multicolumn{6}{|l|}{ Covariates } \\
\hline Seizure & 1798 & 3.4 & 1409 & 2.7 & 0.04 \\
\hline Intellectual disability & 139 & 0.3 & 76 & 0.1 & 0.04 \\
\hline Autism & 309 & 0.6 & 315 & 0.6 & 0.00 \\
\hline Conduct disorder & 286 & 0.5 & 257 & 0.5 & 0.00 \\
\hline Opposition defiant disorder & 29 & 0.1 & 28 & 0.1 & 0.00 \\
\hline Anxiety & $14 \mid 4$ & 2.7 & 1567 & 3 & -0.02 \\
\hline Depression & 757 & 1.4 & 506 & I & 0.04 \\
\hline \multicolumn{6}{|l|}{ Urbanized Level of Residence } \\
\hline I (City) & 17,803 & 33.8 & 17,155 & 32.6 & 0.03 \\
\hline 2 & 25,326 & 48.1 & 25,607 & 48.6 & -0.01 \\
\hline 3 & 6693 & 12.7 & 6762 & 12.8 & 0.00 \\
\hline 4 (Villages) & 2883 & 5.5 & 3181 & 6 & -0.02 \\
\hline
\end{tabular}

Abbreviation: ADHD, attention deficit hyperactivity disorder. 
Table 2 Cox Proportional Hazards Model for Effect of ADHD on Burn Risk

\begin{tabular}{|c|c|c|c|c|c|c|}
\hline \multirow[t]{2}{*}{ Variables } & \multicolumn{3}{|c|}{ Unadjusted Analysis } & \multicolumn{3}{|c|}{ 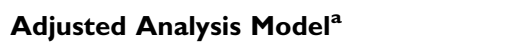 } \\
\hline & HR & $95 \% \mathrm{Cl}$ & $P$ value & HR & $95 \% \mathrm{Cl}$ & $P$ value \\
\hline ADHD & 1.76 & $1.65-1.88$ & $<0.0001$ & 1.78 & $1.66-1.90$ & $<0.0001$ \\
\hline Male gender & 0.97 & $0.90-1.05$ & 0.4539 & 0.98 & $0.91-1.05$ & 0.5278 \\
\hline \multicolumn{7}{|l|}{ Age } \\
\hline $0-5$ (ref.) & 1.00 & & & 1.00 & & \\
\hline $6-11$ & 0.58 & $0.54-0.62$ & $<0.0001$ & 0.57 & $0.54-0.6 \mathrm{I}$ & $<0.0001$ \\
\hline $12-18$ & 0.46 & $0.39-0.54$ & $<0.0001$ & 0.48 & $0.4 I-0.57$ & $<0.0001$ \\
\hline \multicolumn{7}{|l|}{ Urbanized Level of Residence } \\
\hline I (City) (ref.) & 1.00 & & & 1.00 & & \\
\hline 2 & 1.06 & $0.99-1.14$ & 0.1041 & 1.08 & $1.01-1.16$ & 0.0347 \\
\hline 3 & 1.14 & $1.03-1.26$ & 0.0148 & 1.15 & $1.04-1.28$ & $0.007 \mid$ \\
\hline 4(Villages) & 1.20 & $1.05-1.38$ & 0.0091 & 1.25 & $1.08-1.43$ & 0.002 \\
\hline \multicolumn{7}{|l|}{ Covariates } \\
\hline Seizure & 1.28 & $1.10-1.50$ & 0.002 & 1.19 & $1.02-1.40$ & 0.0301 \\
\hline Intellectual disability & 1.50 & $0.91-2.49$ & 0.116 & 1.14 & $0.68-1.89$ & 0.6286 \\
\hline Autism & 0.84 & $0.55-1.28$ & 0.4109 & 0.76 & $0.50-1.15$ & 0.1964 \\
\hline Conduct disorder & 0.95 & $0.62-1.46$ & 0.8147 & 1.07 & $0.70-1.65$ & 0.7604 \\
\hline Opposition defiant disorder & 0.44 & $0.06-3.10$ & 0.4082 & 0.55 & $0.08-3.87$ & 0.5437 \\
\hline Anxiety & 0.65 & $0.52-0.81$ & 0.0002 & 0.77 & $0.6 I-0.98$ & 0.0301 \\
\hline Depression & 0.71 & $0.51-0.99$ & 0.0408 & 0.84 & $0.59-1.20$ & 0.3417 \\
\hline
\end{tabular}

Note: ${ }^{a}$ Adjusted by sex, age, urbanization level of residence, seizure, intellectual disability, autism, conduct disorder, oppositional defiant disorder, anxiety, and depression. Abbreviations: ADHD, attention deficit hyperactivity disorder; $\mathrm{HR}$, hazard ratio; $\mathrm{Cl}$, confidence interval.

Table 3 Effects of Age and Sex on Burn Injury Risk

\begin{tabular}{|c|c|c|c|c|c|c|}
\hline \multirow[t]{2}{*}{ Subgroup } & \multicolumn{3}{|c|}{ Unadjusted Analysis } & \multicolumn{3}{|c|}{ Adjusted Analysis Model ${ }^{\mathbf{a}}$} \\
\hline & HR & $95 \% \mathrm{Cl}$ & $P$ value & HR & $95 \% \mathrm{Cl}$ & $P$ value \\
\hline \multicolumn{7}{|l|}{ Gender } \\
\hline Female & 1.78 & $1.55-2.03$ & $<0.0001$ & 1.80 & $1.58-2.07$ & $<0.0001$ \\
\hline Male & 1.75 & $1.63-1.89$ & $<0.0001$ & 1.77 & $1.64-1.91$ & $<0.0001$ \\
\hline \multicolumn{7}{|l|}{ Age } \\
\hline$<6$ & 1.94 & $1.73-2.17$ & $<0.0001$ & 1.96 & $1.75-2.20$ & $<0.0001$ \\
\hline$\geq 6$ & 1.70 & $1.56-1.84$ & $<0.0001$ & 1.69 & $1.56-1.83$ & $<0.0001$ \\
\hline
\end{tabular}

Note: ${ }^{a}$ Adjusted by sex, age, urbanization level of residence, seizure, intellectual disability, autism, conduct disorder, oppositional defiant disorder, anxiety, and depression. Abbreviations: $\mathrm{HR}$, hazard ratio; $\mathrm{Cl}$, confidence interval.

revealed that the incidence of burn injury in the ADHD group was $10.6 \%$, significantly higher than that in the control group (2\%). To our knowledge, the population included in our study is the largest among related studies. Although our results indicate that children with ADHD were more prone to burn injury, the incidence of burn injury was $4.6 \%$, considerably lower than the rates reported in previous case-control studies. This discrepancy may be attributed to differences in research methodologies. In one previous case-control study, patients were asked to recall burn injuries during an interview; hence, recall bias should be considered. By contrast, the incidence of burn injury was identified in claim records in our study. Cases of minor burn injury may have been concealed if individuals did not seek medical care.

Several population database studies have reported conflicting findings. A database study conducted in Germany compared the risk of hospitalization due to injury between patients aged 3-17 years newly diagnosed as having ADHD and those without ADHD; the results revealed no significant increase in burn risk among patients with ADHD. ${ }^{7}$ However, we noted that confounders were not 
studied in the preceding study, and the heterogeneity of characteristics may have interfered in the evaluation of the influence of ADHD. In another national cohort study, a nationally representative study population of more than 10,000 children aged 5-15 years was selected from a registry database to assess the relationship between unintentional injury and childhood psychiatric disorder. ${ }^{4}$ The results revealed that ADHD was associated with burn injury, but the correlation was dispelled after other psychiatric diseases and psychosocial factors were adjusted for. In the mentioned national cohort study, the study population was aged 5-15 years, which was more limited than the population we explored.

The correlation between ADHD and burn injury has several potential explanations. Impulsive behavior is believed to play a major role in burn injuries. A retrospective study investigated the cause of burn injuries in patients with ADHD and suggested that impulsive behavior contributed to burn injuries in more than $54 \%$ of patients with ADHD. ${ }^{15}$ Risk-seeking behaviors, such as fire-play and fire-setting, are not uncommon among individuals with ADHD. ${ }^{18}$ Additionally, carelessness related to attention deficit, overlooking danger, and impairments in motor coordination and executive function may be associated with burn injuries. ${ }^{18}$ However, limited attention has been paid to these possible mediating factors. Further comprehensive examination of the causal relationship between ADHD and burn injury is warranted.

In this study, individuals diagnosed as having ADHD and aged younger than 6 years were more prone to burn injury. A previous study in Taiwan that analyzed a national database reported a 1.6-fold increase in the risk of burn injury among individuals with ADHD compared with controls for a population aged 6-18 years. ${ }^{5}$ One systematic review investigated the epidemiology of unintentional burn injuries in Asia and revealed that the majority of the injury burden was among younger children, particularly those aged younger than 5 years. ${ }^{22}$ The effect of age on burn injury among children with ADHD required urgent clarification. Our findings indicate that individuals with ADHD and who were aged younger than 6 years were at higher risk of burn injury. These higher numbers of burns in early childhood may be linked to the inquisitive behavior of children who have not acquired sufficient experience regarding dangers as well as their total dependency on parents and caregivers. ${ }^{31}$ The findings highlight the need to establish policy for the prevention of burn injury among children with ADHD.
This study has several strengths. First, the nationally representative study population was large, and selection bias was minimized. Second, individuals with ADHD were identified by physician diagnoses rather than through a self-report scale, preventing recall bias. Furthermore, we used propensity score matching, which provided a "least biased" approach to measuring the influence of ADHD on burn injury risk.

This study also has several limitations. First, diagnoses of ADHD and diseases were obtained from administrative claims data; thus, possible misclassifications may exist due to help-seeking behavior. Stigma regarding mental illness may prevent individuals from visiting a psychiatric clinic. Second, we selected individuals by using Taiwan's NHIRD and records from January 1996 to December 2013. The data registered during the period between 1996 and 2013 were recorded using ICD-9-CM, which had more strict criteria regarding ADHD than ICD-11. Cases of ADHD may be missing due to this methodological limitation. An updated diagnostic system should be considered in future analysis. Third, some vital clinical information, such as socioeconomical status, parental supervision, family history, and lifestyle, was not obtained from the claims data. These psychosocial confounding factors may have significant influences on burn risk, particularly for young children. Finally, the severity and predominant features of ADHD symptoms were not assessed, and data for the analysis of the correlation of ADHD symptoms with burn injury severity were not available. Further comprehensive research should be conducted to analyze the relationship between ADHD symptoms and burn severity.

\section{Conclusion}

The results of this study suggest that clinicians pay attention to burn risk for patients with ADHD, particularly for children aged younger than 6 years. Continual efforts are required to enhance public awareness of burn risk for young children with ADHD. Further exploration of the causal relationship between ADHD and burn injury will provide more information for the prevention of burn injury.

\section{Acknowledgments}

The authors thank the staff of the Health Information and Epidemiology Laboratory (CLRPG6G0042, CLRPG6G0 043) for their comments and assistance with the data analysis.

\section{Disclosure}

On behalf of all authors, the corresponding author states that there is no conflict of interest. 


\section{References}

1. Thomas R, Sanders S, Doust J, Beller E, Glasziou P. Prevalence of attention-deficit/hyperactivity disorder: a systematic review and meta-analysis. Pediatrics. 2015;135(4):e994-1001. doi:10.1542/peds. 2014-3482

2. Swanson JM. Role of executive function in ADHD. $J$ Clin Psychiatry. 2003;64 Suppl 14(Suppl 14):35-39.

3. Chang Z, Lichtenstein P, D'Onofrio BM, Sjolander A, Larsson H. Serious transport accidents in adults with attention-deficit/hyperactivity disorder and the effect of medication: a population-based study. JAMA Psychiatry. 2014;71(3):319-325. doi:10.1001/jamapsychiatry.2013.4174

4. Rowe R, Maughan B, Goodman R. Childhood psychiatric disorder and unintentional injury: findings from a national cohort study J Pediatr Psychol. 2004;29(2):119-130. doi:10.1093/jpepsy/jsh015

5. Tai YM, Gau SS, Gau CS. Injury-proneness of youth with attention-deficit hyperactivity disorder: a national clinical data analysis in Taiwan. Res Dev Disabil. 2013;34(3):1100-1108. doi:10.1016/ j.ridd.2012.11.027

6. Silva D, Colvin L, Hagemann E, Stanley F, Bower C. Children diagnosed with attention deficit disorder and their hospitalisations: population data linkage study. Eur Child Adolesc Psychiatry. 2014;23 (11):1043-1050. doi:10.1007/s00787-014-0545-8

7. Lindemann C, Langner I, Banaschewski T, Garbe E, Mikolajczyk RT. The risk of hospitalizations with injury diagnoses in a matched cohor of children and adolescents with and without attention deficit/hyperactivity disorder in Germany: a database study. Front Pediatr. 2017;5:220. doi:10.3389/fped.2017.00220

8. Chang Z, Ghirardi L, Quinn PD, Asherson P, D'Onofrio BM, Larsson H. Risks and benefits of attention-deficit/hyperactivity disorder medication on behavioral and neuropsychiatric outcomes: a qualitative review of pharmacoepidemiology studies using linked prescription databases. Biol Psychiatry. 2019;86(5):335-343. doi:10. 1016/j.biopsych.2019.04.009

9. Bruce B, Kirkland S, Waschbusch D. The relationship between childhood behaviour disorders and unintentional injury events. Paediatr Child Health. 2007;12(9):749-754. doi:10.1093/pch/12.9.749

10. DiScala C, Lescohier I, Barthel M, Li G. Injuries to children with attention deficit hyperactivity disorder. Pediatrics. 1998;102 (6):1415-1421. doi:10.1542/peds.102.6.1415

11. Dalsgaard S, Ostergaard SD, Leckman JF, Mortensen PB, Pedersen MG. Mortality in children, adolescents, and adults with attention deficit hyperactivity disorder: a nationwide cohort study. Lancet (London, England). 2015;385(9983):2190-2196. doi:10.10 16/S0140-6736(14)61684-6

12. Chen VC, Chan HL, Wu SI, et al. Attention-deficit/hyperactivity disorder and mortality risk in Taiwan. JAMA. 2019;2(8):e198714. doi:10.1001/jamanetworkopen.2019.8714

13. Noronha DO, Faust J. Identifying the variables impacting post-burn psychological adjustment: a meta-analysis. $J$ Pediatr Psychol. 2006;32(3):380-391. doi:10.1093/jpepsy/js1014

14. Chen SH, Chen YC, Chen TJ, Ma H. Epidemiology of burns in Taiwan: a nationwide report including inpatients and outpatients. Burns. 2014;40(7):1397-1405. doi:10.1016/j.burns.2014.01.014
15. Thomas CR, Ayoub M, Rosenberg L, Robert RS, Meyer WJ. Attention deficit hyperactivity disorder \& pediatric burn injury: a preliminary retrospective study. Burns. 2004;30(3):221-223. doi:10.1016/j.burns.2003.10.013

16. Badger K, Anderson L, Kagan RJ. Attention deficit-hyperactivity disorder in children with burn injuries. J Burn Care Res. 2008;29 (5):724-729. doi:10.1097/BCR.0b013e31818480e1

17. Mangus RS, Bergman D, Zieger M, Coleman JJ. Burn injuries in children with attention-deficit/hyperactivity disorder. Burns. 2004;30 (2):148-150. doi:10.1016/j.burns.2003.09.020

18. Fritz KM, Butz C. Attention deficit/hyperactivity disorder and pediatric burn injury: important considerations regarding premorbid risk. Curr Opin Pediatr. 2007;19(5):565-569. doi:10.1097/MOP.0b013e3282ef498c

19. Ghanizadeh A. Small burns among out-patient children and adolescents with attention deficit hyperactivity disorder. Burns. 2008;34 (4):546-548. doi:10.1016/j.burns.2007.07.015

20. Swensen A, Birnbaum HG, Ben Hamadi R, Greenberg P, Cremieux PY, Secnik K. Incidence and costs of accidents among attention-deficit/hyperactivity disorder patients. $J$ Adolesc Health. 2004;35(4):346.e341-349. doi:10.1016/j.jadohealth.2003.12.003

21. Emond A, Sheahan C, Mytton J, Hollen L. Developmental and behavioural associations of burns and scalds in children: a prospective population-based study. Arch Dis Child. 2017;102 (5):428-483. doi:10.1136/archdischild-2016-311644

22. Golshan A, Patel C, Hyder AA. A systematic review of the epidemiology of unintentional burn injuries in South Asia. J Public Health (Oxford, England). 2013;35(3):384-396. doi:10.1093/pubmed/fds102

23. Little RJ, Rubin DB. Causal effects in clinical and epidemiological studies via potential outcomes: concepts and analytical approaches. Annu Rev Public Health. 2000;21(1):121-145. doi:10.1146/annurev. publhealth.21.1.121

24. Austin PC. The use of propensity score methods with survival or time-to-event outcomes: reporting measures of effect similar to those used in randomized experiments. Stat Med. 2014;33(7):1242-1258. doi:10.1002/sim.5984

25. Institutes NHR. Introduction to the National Health Insurance Research Database (NHIRD). Taiwan; 2013.

26. Hsieh CY, Su CC, Shao SC, et al. Taiwan's National Health Insurance Research Database: past and future. Clin Epidemiol. 2019;11: 349-358. doi:10.2147/CLEP.S196293

27. Lin LY, Warren-Gash C, Smeeth L, Chen PC. Data resource profile: the National Health Insurance Research Database (NHIRD) Epidemiol Health. 2018;40:e2018062. doi:10.4178/epih.e2018062

28. Chen VC, Wu SI, Huang KY, et al. Herpes zoster and dementia: a nationwide population-based cohort study. J Clin Psychiatry. 2018;79(1).

29. Lee MJ, Lee SY, Yuan SS, et al. Comorbidity of narcolepsy and depressive disorders: a nationwide population-based study in Taiwan. Sleep Med. 2017;39:95-100. doi:10.1016/j.sleep.2017.07.022

30. Boschini LP, Tyson AF, Samuel JC, et al. The role of seizure disorders in burn injury and outcome in Sub-Saharan Africa. J Burn Care Res. 2014;35 (6):e406-412. doi:10.1097/BCR.0000000000000026

31. Othman N, Kendrick D. Epidemiology of burn injuries in the East Mediterranean Region: a systematic review. BMC Public Health. 2010;10(1):83. doi:10.1186/1471-2458-10-83
Neuropsychiatric Disease and Treatment

\section{Publish your work in this journal}

Neuropsychiatric Disease and Treatment is an international, peerreviewed journal of clinical therapeutics and pharmacology focusing on concise rapid reporting of clinical or pre-clinical studies on a range of neuropsychiatric and neurological disorders. This journal is indexed on PubMed Central, the 'PsycINFO' database and CAS, an is the official journal of The International Neuropsychiatric Association (INA). The manuscript management system is completely online and includes a very quick and fair peer-review system, which is all easy to use. Visit http://www.dovepress.com/testimonials.php to read real quotes from published authors. 